\title{
Optimization of 7,12-dimethylbenz(a)anthracene-induced rat epithelial ovarian tumors
}

\author{
XIU YING YANG ${ }^{1}$, YING LI $^{1}$, SONG QI CAI ${ }^{2}$, LI WANG $^{3}$ and JIN WEI QIANG ${ }^{1}$ \\ ${ }^{1}$ Department of Radiology, Jinshan Hospital, Fudan University, Shanghai 201508; \\ ${ }^{2}$ Departments of Radiology, Zhongshan Hospital, Fudan University, Shanghai 200032; \\ ${ }^{3}$ Department of Pathology, Jinshan Hospital, Fudan University, Shanghai 201508, P.R. China
}

Received May 19, 2020; Accepted November 13, 2020

DOI: $10.3892 / 01.2021 .12467$

\begin{abstract}
Ovarian carcinoma is the second most common malignant tumor of the female reproductive system and an notable cause of cancer death. The detection and diagnosis of early ovarian carcinomas are still clinical challenges, which calls for imaging studies using early ovarian carcinoma animal models. The present study aimed to optimize the 7,12-dimethylbenz(a)anthracene (DMBA)-induced model of rat ovarian tumors by investigating the delivery methods, induction dose and time of DMBA exposure, and explored the morphological features of tumors using MRI. Three schemes were performed. In scheme one the ovary was covered with absorbable hemostatic gauze loaded with a high concentration of liquid DMBA. For this scheme, 150 Sprague-Dawley rats were divided into three groups depending on the DMBA dose (1.0, 2.0 and $3.0 \mathrm{mg})$. In scheme two DMBA solution was injected under the ovarian capsule. For this scheme, 159 rats were divided into $0.5,1.0$ and $1.5 \mathrm{mg}$ DMBA groups. In scheme three the ovary was covered with absorbable gauze loaded with a high concentration of solid DMBA. For this scheme 161 rats were divided into 1.0, 2.0 and 3.0 mg DMBA groups. Each group of the three schemes was further subdivided into 60-, 90-, 120-, 150- and 180-day groups. In scheme two, the tumor formation rate was $75.6 \%(99 / 131)$, which was the highest in the $1.5 \mathrm{mg}$ group $(86.4 \%, 38 / 44)$ and reached $100 \%(10 / 10)$ on day 120 . The induced tumors were serous in $93.9 \%(93 / 99)$ of tumors. Borderline ovarian tumors accounted for 19.2\% (19/99) of all tumors, and ovarian cancer accounted for $46.5 \%$ (46/99). The mean maximum diameter (MMD) of borderline ovarian tumors was $10.29 \pm 3.41 \mathrm{~mm}$, and that of ovarian cancer was $15.19 \pm 7.10 \mathrm{~mm}$. MMD of the solid components increased with
\end{abstract}

Correspondence to: Professor Jin Wei Qiang, Department of Radiology, Jinshan Hospital, Fudan University, 1508 Longhang Road, Jinshan, Shanghai 201508, P.R. China

E-mail: dr.jinweiqiang@163.com

Key words: animal model, 7,12-dimethylbenz(a)anthracene, ovarian tumor, borderline ovarian tumor, ovarian carcinoma, Sprague-Dawley rat increasing malignancy. Cystic, cystic-solid and solid tumors were observed. The ovarian subcapsular injection of $1.5 \mathrm{mg}$ DMBA was the best scheme for the rat ovarian tumor model. The present model is ideal for investigating the occurrence, development and imaging of ovarian tumors.

\section{Introduction}

Ovarian carcinomas are a group of malignant tumors, whose mortality rate ranks second in gynecological tumors worldwide (1). Ovarian tumors can be divided into ovarian carcinomas, borderline tumors and benign tumors according to their biological behavior and histological differentiation (2). Ovarian carcinomas can also be divided into five main histological types (high grade serous, endometrioid, clear cell, mucinous and low grade serous ovarian carcinomas) according to different histological epithelia (3). Currently, high-grade serous carcinomas (HGSC) and low-grade serous carcinomas (LGSC) are considered to be two distinct tumors. HGSC does not develop from well-differentiated LGSC and likely arises from the fallopian tube epithelium, with an obvious mitotic activity, nuclear atypia and common TP53 mutations (4-6). Meanwhile, LGSC shows low mitotic activity, nuclear atypia and frequent KRAS and BRAF mutations $(7,8)$. Due to its late detection, the survival rate of patients with ovarian carcinomas is low. The 5-year survival rate is only $\sim 29 \%$ for patients with advanced stage (III and IV combined) but is $>92 \%$ for patients with stage I carcinoma $(9,10)$. Therefore, the early detection and accurate diagnosis of ovarian carcinomas may improve the patient's survival rate and quality of life. Unfortunately, due to a lack of effective imaging tools or biomarkers for screening early ovarian carcinomas, it is difficult to conduct a comprehensive imaging study for early ovarian carcinomas (11). A good animal model of ovarian precancerous lesions, borderline tumors and early carcinomas will be helpful for investigating the occurrence, development and imaging of ovarian carcinomas.

Chemically induced animal models of ovarian tumors can exhibit oncogenesis, development, invasion, and metastasis (12). 7,12-dimethylbenz[a]anthracene (DMBA), a frequently used carcinogen to induce ovarian tumors, has been confirmed to have specificity for inducing ovarian adenocarcinoma (13-16). Studies have shown that DMBA-induced oncogenes in rat ovarian adenocarcinomas were similar to 
those in human ovarian adenocarcinomas $(14,16)$. However, previous researchers used non-absorbable materials to load chemical carcinogens (14). The induced tumors were accompanied by inflammatory granulomas and were mostly advanced ovarian carcinomas, which are not suitable for the investigation of borderline ovarian tumors and early ovarian carcinomas. Therefore, the present study aimed to optimize DMBA induction schemes for rat borderline ovarian tumors and early ovarian carcinomas by comparing different delivery methods, induction doses and times.

\section{Materials and methods}

Ethics. The study was approved by The Institutional Review Board of Jinshan Hospital, Fudan University (Shanghai, China), and all procedures involving animal studies were in accordance with the Guide for the Care and Use of Laboratory Animals of the National Science and Technology Committee of China. During the experimental process, rats were euthanized when they developed cachexia or abnormally dilated abdominal cavity.

Animal breeding. In total, 500 female Sprague-Dawley rats weighing $150-200 \mathrm{~g}$, with ages ranging from 5 to 7 weeks [Shanghai Experimental Animal Co., Ltd., SCXK(SH)2012-0006] were fed for one week before surgery. The rats were maintained in a room under a temperature of $22 \pm 2^{\circ} \mathrm{C}$ with a $12-12 \mathrm{~h}$ light/dark cycle. Food and deionized water were available ad libitum.

Experimental grouping. The current study was performed by using three experimental schemes of DMBA delivery and corresponding control groups. Scheme one included 150 experimental rats divided into three groups of 50 rats per group according to three different doses (1.0, 2.0 and $3.0 \mathrm{mg})$. Scheme two included 159 rats divided into $0.5,1.0$ and $1.5 \mathrm{mg}$ groups, with 51, 53 and 55 rats in each group, respectively. Scheme three included 161 rats divided into 1.0,2.0 and $3.0 \mathrm{mg}$ groups, with 50,53 and 58 rats in each group, respectively. Rats of different dose groups in each scheme were subdivided into five groups according to the time of DMBA exposure $(60,90$, 120,150 and 180 days). In total, 30 control rats were divided into three groups according to the corresponding experimental schemes, with 10 rats in each group.

DMBA preparation. For scheme one DMBA (99\% purity; Sigma-Aldrich; Merck KGaA) was dissolved in DMSO solvent (analytical pure; Shanghai Shenggong Biology Engineering Technology Service, Ltd.) to produce 1.0,2.0 and 3.0 mg DMBA per $0.02 \mathrm{ml}$ solution. A piece of $0.6 \mathrm{x} 0.6 \mathrm{~mm}$ sterile absorbable gauze (Danatai; Yunnan Dehua Biological Pharmaceutical Corporation) was folded twice to make its length and width $0.3 \times 0.3 \mathrm{~mm}$. The prepared DMBA solution was injected into absorbable gauze slowly with a microsyringe. For scheme two DMBA was dissolved in DMSO solvent to produce a DMBA content per $0.02 \mathrm{ml}$ solution of $0.5,1.0$ and $1.5 \mathrm{mg}$. For scheme three DMBA was heated to a melting point of $124^{\circ} \mathrm{C}$. Absorbable hemostatic gauze was immersed in melted DMBA and contained 1.0, 2.0 and $3.0 \mathrm{mg}$ of carcinogen, as weighed on a microchemical balance.
$D M B A$ exposure to the ovary. Ovaries were exposed to DMBA as described in a previous study (17). Rats were anesthetized by intraperitoneal injection of $2 \%$ pentobarbital sodium at $50 \mathrm{mg} / \mathrm{kg}$. A transverse, $1.5-\mathrm{cm}$ mid-lumbar incision was made in the right flank of the animal, $5 \mathrm{~mm}$ ventral to the lumbar muscles. Ovaries and fat pads were surgically exposed. For scheme one the ovary was covered with absorbable hemostatic gauze loaded with a high concentration of liquid DMBA or DMSO (serving as the control), wrapped with periovarian fat and sealed with human absorbable fibrin glue (Hualan Biological Co., Ltd.) (Fig. 1). For scheme two DMBA solution or DMSO (serving as the control) was injected under the ovarian capsule, and the pinholes were sealed with absorbable fibrin glue (Fig. 1). For scheme three the ovary was covered with absorbable gauze loaded with a high concentration of solid DMBA or absorbable gauze only (serving as the control) and wrapped with periovarian fat (Fig. 1). An antibiotic ( $10^{5}$ units of benzylpenicillin potassium) was administered intraperitoneally for prophylaxis against infection before the abdominal wall was closed.

MRI. After anesthesia with $2 \%$ pentobarbital sodium at $50 \mathrm{mg} / \mathrm{kg}$, all rats underwent MRI, which was performed as described in a previous study (18). On MR images, the tumor configurations were classified into cystic, cystic-solid and solid according to their gross morphology (19). The maximum diameter (MMD) of the tumors and solid components and the thickness of the wall and septum were measured.

Histopathological analyses. Rats were anesthetized with a single intraperitoneal injection of $2 \%$ sodium pentobarbital $(50 \mathrm{mg} / \mathrm{kg})$ and then euthanized by cervical dislocation. Death was confirmed by checking breathing and heartbeat. Verification of death was supplemented by percutaneous cardiac puncture before tissues were collected. Reproductive system organs and abnormal morphological tissues were removed. The specimens were cut into $3-\mu \mathrm{m}$ sections for hematoxylin and eosin staining by a pathologist (LW, with 18 years of experience in human and murine gynecological pathology). Staining steps are as follows. Tissues were immersed in $10 \%$ (v/v) neutral buffered formalin for $48 \mathrm{~h}$ at room temperature, then were embedded in paraffin. Sections were dewaxed at $60^{\circ} \mathrm{C}$ for $20 \mathrm{~min}$, following washing with xylene twice, each for $15 \mathrm{~min}$. Sections were hydrated with $100 \%$ absolute ethanol for $2 \mathrm{~min}, 95 \%$ ethanol for $1 \mathrm{~min}$, $80 \%$ ethanol for $1 \mathrm{~min}, 75 \%$ ethanol for $1 \mathrm{~min}$ then washed with distilled water for $2 \mathrm{~min}$. Hematoxylin staining was performed at room temperature for $5 \mathrm{~min}$ and then sections were washed with running water. Eosin staining was performed at room temperature for $2 \mathrm{~min}$. The histopathological analysis was performed under a light microscope with magnification $\mathrm{x} 200$. According to the histopathological characteristics of the cells, the ovarian tumors were divided into benign, borderline and malignant (2).

Statistical analyses. Statistical analyses were performed with SPSS version 22.0 (IBM Corp.). The mortality rate and tumor formation rate of rats were compared using $\chi^{2}$ for multiple groups, and the pairwise comparison used the partitions of the $\chi^{2}$ method. Differences in the MMD of the tumors and solid components and the thickness of the wall and septum 
Table I. Mortality rate of rats in different 7,12-dimethylbenz(a)anthracene-induced schemes.

\begin{tabular}{|c|c|c|c|c|c|}
\hline $\begin{array}{l}\text { Scheme } 1 \text { dose, } \\
\text { mg }\end{array}$ & $\begin{array}{c}\text { Mortality rate, } \\
\text { n/total }(\%)\end{array}$ & $\begin{array}{c}\text { Scheme } 2 \text { dose, } \\
\text { mg }\end{array}$ & $\begin{array}{l}\text { Mortality rate, } \\
\text { n/total }(\%)\end{array}$ & $\begin{array}{c}\text { Scheme } 3 \text { dose, } \\
\text { mg }\end{array}$ & $\begin{array}{c}\text { Mortality rate, } \\
\text { n/total }(\%)\end{array}$ \\
\hline 1.0 & $23 / 50 \quad(46.0)$ & 0.5 & $9 / 51 \quad(17.6)$ & 1.0 & $7 / 50 \quad(14.0)$ \\
\hline 2.0 & $43 / 50 \quad(86.0)$ & 1.0 & $8 / 53 \quad(15.1)$ & 2.0 & $11 / 53 \quad(20.8)$ \\
\hline 3.0 & $43 / 50 \quad(86.0)$ & 1.5 & $11 / 55 \quad(20.0)$ & 3.0 & $20 / 58 \quad(34.5)$ \\
\hline Total & $109 / 150(72.7)$ & Total & 28/159 (17.6) & Total & $38 / 161(23.6)$ \\
\hline $0^{\mathrm{a}}$ & $1 / 10 \quad(10.0)$ & $0^{\mathrm{a}}$ & $0 / 10 \quad(0.0)$ & 0 & $2 / 10 \quad(20.0)$ \\
\hline
\end{tabular}

${ }^{\mathrm{a} C o n t r o l ~ g r o u p .}$

Table II. The mortality rate and tumor formation rate of rats in three schemes.

\begin{tabular}{lcccrrrr}
\hline Rate & Scheme one & Scheme Two & Scheme Three & P-value & $\mathrm{P}_{1}$ & $\mathrm{P}_{2}$ & $\mathrm{P}_{3}$ \\
\hline Mortality rate, $\%$ & 72.7 & 17.6 & 23.6 & $<0.0001$ & $<0.05$ & $<0.05$ & $>0.05$ \\
Formation rate, $\%$ & 78.0 & 75.6 & 87.8 & 0.0400 & $>0.05$ & $>0.05$ & $<0.05$ \\
\hline
\end{tabular}

$\mathrm{P}_{1}$, scheme one vs. two. $\mathrm{P}_{2}$ one vs. three. $\mathrm{P}_{3}$ two vs. three.

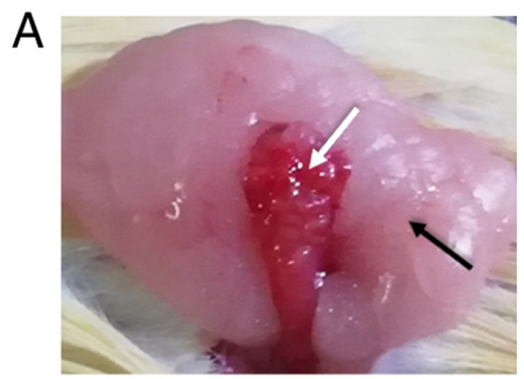

B
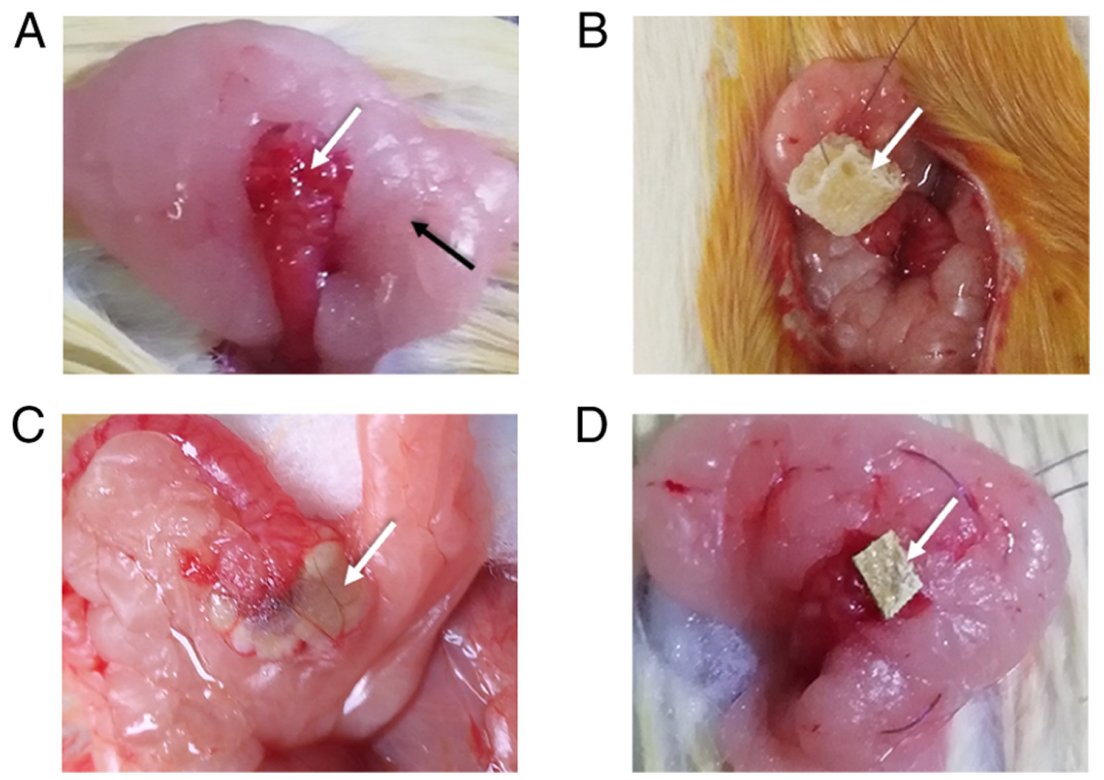

Figure 1. DMBA exposure to the ovary. (A) Ovary of a Sprague-Dawley rat (white arrow) and fat around the ovary (black arrow). (B) Absorbable gauze carrying liquid DMBA (white arrow) was fixed to the ovarian surface in scheme one. (C) DMBA solution is injected under the ovarian capsule (white arrow) in scheme two. (D) Absorbable hemostatic gauze carrying DMBA. Solid DMBA was fixed to the ovarian surface in scheme three (white arrow). DMBA, 7,12-dimethylbenz(a)anthracene.

between the three groups were compared using one-way ANOVA followed by Fisher's Least Significant Difference post hoc, or Kruskal-Wallis followed by Dunn's post hoc were used as appropriate. Spearman's rank correlation analysis was used to evaluate the correlation between dose, time and tumor differentiation. $\mathrm{P}<0.05$ was considered to indicate a statistically significant difference. Fisher's test was performed to compare differences in tumor configurations between groups. All variables are expressed as the mean value \pm standard deviation, unless otherwise shown.

\section{Results}

Mortality rate of rats. The mortality rates of rats in different experimental groups and the control group are summarized in Tables I and II. In scheme one, the overall mortality rate of rats was $72.7 \%(109 / 150)$ in the experimental group and it was $46.0 \%$ (23/50) in the $1.0 \mathrm{mg}$ group. Most dead rats had a markedly dilated bowel, which was considered intestinal obstruction. Ovarian and intestinal adhesions were visible in only a few rats. In the corresponding control group, the mortality rate was 
Table III. Tumor formation rate in different dose and time groups in scheme one.

A, $1.0 \mathrm{mg}$ dose

\begin{tabular}{|c|c|c|c|c|}
\hline Time, days & Total, n/total (\%) & BT, n/total (\%) & BOT, n/total (\%) & OCA, n/total (\%) \\
\hline 60 & $0 / 2 \quad(0.0)$ & $0 / 2 \quad(0.0)$ & $(0.0)$ & $(0.0)$ \\
\hline 90 & $1 / 2 \quad(50.0)$ & $1 / 2 \quad(50.0)$ & $(0.0)$ & $(0.0)$ \\
\hline 120 & $3 / 3 \quad(100.0)$ & $2 / 3 \quad(66.7)$ & $(0.0)$ & $(33.3)$ \\
\hline 150 & $8 / 10(80.0)$ & $2 / 10(20.0)$ & $1 / 10(10.0)$ & $5 / 10 \quad(50.0)$ \\
\hline 180 & $8 / 10(80.0)$ & $0 / 10 \quad(0.0)$ & $0 / 10 \quad(0.0)$ & $8 / 10 \quad(80.0)$ \\
\hline Total & 20/27 (74.1) & $5 / 27(18.5)$ & $1 / 27 \quad(3.7)$ & $14 / 27$ (51.9) \\
\hline
\end{tabular}

$\mathrm{B}, 2.0 \mathrm{mg}$ dose

\begin{tabular}{|c|c|c|c|c|}
\hline Time, days & Total, n/total (\%) & BT, n/total (\%) & BOT, n/total (\%) & OCA, n/total (\%) \\
\hline 60 & $0 / 1 \quad(0.0)$ & $0 / 1 \quad(0.0)$ & $0 / 1 \quad(0.0)$ & $0 / 1 \quad(0.0)$ \\
\hline 90 & $1 / 1(100.0)$ & $1 / 1(100.0)$ & $0 / 1 \quad(0.0)$ & $0 / 1 \quad(0.0)$ \\
\hline 120 & $5 / 5(100.0)$ & $1 / 5 \quad(20.0)$ & $3 / 5(60.0)$ & $1 / 5(20.0)$ \\
\hline 150 & $0 / 0 \quad(0.0)$ & $0 / 0 \quad(0.0)$ & $0 / 0 \quad(0.0)$ & $0 / 0 \quad(0.0)$ \\
\hline 180 & $(0.0)$ & $(0.0)$ & $0 / 0 \quad(0.0)$ & $0 / 0 \quad(0.0)$ \\
\hline Total & $6 / 7 \quad(85.7)$ & $2 / 7 \quad(28.6)$ & $3 / 7(42.9)$ & $1 / 7(14.3)$ \\
\hline
\end{tabular}

C, $3.0 \mathrm{mg}$ dose

\begin{tabular}{lcccr}
\hline Time, days & Total, n/total $(\%)$ & BT, n/total $(\%)$ & BOT, n/total $(\%)$ & OCA, n/total $(\%)$ \\
\hline 60 & $1 / 2(50.0)$ & $1 / 2(50.0)$ & $0 / 2(0.0)$ & $0 / 2(0.0)$ \\
90 & $2 / 2(100.0)$ & $1 / 2(50.0)$ & $1 / 2(50.0)$ & $0 / 2(0.0)$ \\
120 & $3 / 3(100.0)$ & $0 / 3(0.0)$ & $1 / 3(33.3)$ & $2 / 3(66.7)$ \\
150 & $0 / 0 \quad(0.0)$ & $0 / 0(0.0)$ & $0 / 0(0.0)$ & $0 / 0(0.0)$ \\
180 & $0 / 0 \quad(0.0)$ & $0 / 0(0.0)$ & $0 / 0(0.0)$ & $0 / 0(0.0)$ \\
Total & $6 / 7(85.7)$ & $2 / 7(28.6)$ & $2 / 7(28.6)$ & $2 / 7(28.6)$ \\
\hline
\end{tabular}

$\mathrm{D}, 0 \mathrm{mg}$ dose

\begin{tabular}{lcccc}
\hline Time, days & Total, n/total $(\%)$ & BT, n/total $(\%)$ & BOT, n/total $(\%)$ & OCA, n/total $(\%)$ \\
\hline 180 & $0 / 9(0.0)$ & $0 / 9(0.0)$ & $0 / 9(0.0)$ & $0 / 9(0.0)$ \\
\hline
\end{tabular}

BT, benign ovarian tumors; BOT, borderline ovarian tumors; OCA, ovarian carcinomas.

$10.0 \%(1 / 10)$. In scheme two, the overall mortality rate of rats was $17.6 \%(28 / 159)$ in the experimental group and no rats died (0/10) in the control group. In scheme three, 123 rats survived, with a mortality rate of $23.6 \%(38 / 161)$ in the experimental group (Table II). The mortality rate was only $14.0 \%$ (7/50) for the $1.0 \mathrm{mg}$ group, but it was $34.5 \%$ (20/58) for the $3.0 \mathrm{mg}$ group. Most of the rats died in the late stage of the experiment. The tumor adhered to the surrounding tissues, and bloody ascites was found in nine rats. Two rats died in the control group in scheme three. All the dead rats in the control groups had a markedly dilated bowel, indicative of intestinal obstruction. As shown in Table I, the mortality rates gradually increased in all three experimental groups with increasing DMBA doses.
Incidence of ovarian neoplasia and histopathology results. The incidence of ovarian neoplasia and histopathology results are listed in Tables II-V. As shown in scheme one of Tables II and III, 32/41 rats developed ovarian tumors, and the overall tumor formation rate was $78.0 \%$ (32/41). There were nine cystadenomas, six borderline tumors and 17 ovarian carcinomas (five LGSC and 12 HGSC) (Figs. 2 and 3), all of which were serous tumors. Both benign and borderline tumors were cystic, and ovarian carcinomas were cystic (1/17, 5.9\%), cystic-solid (14/17, 82.4\%) and solid (2/17, 11.8\%) (Fig. 4).

As seen in scheme two of Tables II and IV, the overall tumor formation rate was $75.6 \%(99 / 131)$, which is close to the $0.5 \mathrm{mg}$ group in the preliminary experiment $(75 \%, 15 / 20)$. 
Table IV. Tumor formation rate in different dose and time groups in scheme two.

A, $0.5 \mathrm{mg}$ dose

\begin{tabular}{lcccr}
\hline Time, days & Total, n/total $(\%)$ & BT, n/total $(\%)$ & BOT, n/total $(\%)$ & OCA, n/total $(\%)$ \\
\hline 60 & $1 / 6(16.7)$ & $1 / 6(16.7)$ & $0 / 6(0.0)$ & $0 / 6 \quad(0.0)$ \\
90 & $8 / 10(80.0)$ & $6 / 10(60.0)$ & $2 / 10(20.0)$ & $0 / 10(0.0)$ \\
$120^{\mathrm{a}}$ & $4 / 6(66.7)$ & $4 / 6(66.7)$ & $0 / 6(0.0)$ & $0 / 6$ \\
150 & $8 / 10(80.0)$ & $2 / 10(20.0)$ & $1 / 10(10.0)$ & $5 / 10(50.0)$ \\
180 & $7 / 10(70.0)$ & $2 / 10(20.0)$ & $0 / 10(0.0)$ & $5 / 10(50.0)$ \\
Total & $28 / 42(66.7)$ & $15 / 42(35.7)$ & $3 / 42(7.1)$ & $10 / 42(23.8)$ \\
\hline
\end{tabular}

$\mathrm{B}, 1.0 \mathrm{mg}$ dose

\begin{tabular}{|c|c|c|c|c|}
\hline Time, days & Total, n/total (\%) & BT, n/total (\%) & BOT, n/total (\%) & OCA, n/total (\%) \\
\hline 60 & $2 / 7 \quad(28.6)$ & $1 / 7 \quad(14.3)$ & $1 / 7 \quad(14.3)$ & $0 / 7 \quad(0.0)$ \\
\hline 90 & $8 / 10 \quad(80.0)$ & 4/10 (40.0) & $3 / 10(30.0)$ & $1 / 10(10.0)$ \\
\hline $120^{\mathrm{a}}$ & $5 / 10 \quad(50.0)$ & $2 / 10 \quad(20.0)$ & $1 / 10 \quad(10.0)$ & $2 / 10(20.0)$ \\
\hline 150 & $8 / 8 \quad(100.0)$ & $1 / 8 \quad(12.5)$ & $1 / 8 \quad(12.5)$ & $6 / 8 \quad(75.0)$ \\
\hline 180 & $10 / 10(100.0)$ & $3 / 10(30.0)$ & $0 / 10 \quad(0.0)$ & $7 / 10(70.0)$ \\
\hline Total & $33 / 45(73.3)$ & $11 / 45(24.4)$ & $6 / 45(13.3)$ & $16 / 45(35.6)$ \\
\hline
\end{tabular}

C, $1.5 \mathrm{mg}$ dose $\mathrm{b}^{\mathrm{b}}$

\begin{tabular}{|c|c|c|c|c|}
\hline Time, days & Total, n/total (\%) & BT, n/total (\%) & BOT, n/total (\%) & OCA, n/total (\%) \\
\hline 60 & $6 / 6 \quad(100.0)$ & $2 / 6 \quad(33.3)$ & $4 / 6 \quad(66.7)$ & $(0.0)$ \\
\hline 90 & $7 / 8 \quad(87.5)$ & $2 / 8 \quad(25.0)$ & $2 / 8 \quad(25.0)$ & $3 / 8 \quad(37.5)$ \\
\hline $120^{\mathrm{a}}$ & $10 / 10(100.0)$ & $2 / 10(20.0)$ & $3 / 10(30.0)$ & $5 / 10(50.0)$ \\
\hline 150 & $8 / 10 \quad(80.0)$ & $2 / 10(20.0)$ & $1 / 10(10.0)$ & $5 / 10(50.0)$ \\
\hline 180 & $7 / 10 \quad(70.0)$ & $0 / 10 \quad(0.0)$ & $0 / 10 \quad(0.0)$ & $7 / 10(70.0)$ \\
\hline Total & $38 / 44 \quad(86.4)$ & 8/44 (18.2) & $10 / 44(22.7)$ & $20 / 44(45.5)$ \\
\hline
\end{tabular}

$\mathrm{D}, 0 \mathrm{mg}$ dose

\begin{tabular}{lcccc}
\hline Time, days & Total, n/total $(\%)$ & BT, n/total $(\%)$ & BOT, n/total $(\%)$ & OCA, n/total $(\%)$ \\
\hline 180 & $0 / 10(0.0)$ & $0 / 10(0.0)$ & $0 / 10(0.0)$ & $0 / 10(0.0)$
\end{tabular}

${ }^{\mathrm{a}}$ Ovarian tumor differentiation positively correlated with the dose $(\rho=0.523, \mathrm{P}=0.022) .{ }^{\mathrm{b}}$ Ovarian tumor differentiation positively correlated with the induction time $(\rho=0.506, \mathrm{P}=0.001)$. BT, benign ovarian tumors; BOT, borderline ovarian tumors; OCA, ovarian carcinomas .

Of 99 tumors, 34 were benign, 19 were borderline and 46 were ovarian carcinomas (11 LGSC and 35 HGSC) (Figs. 2 and 3); 93 were serous tumors, four were endometrioid tumors, one was a seromucinous tumor and one was a mucinous tumor. All benign and borderline tumors were also cystic. Ovarian carcinomas were cystic (8/46, 17.4\%), cystic-solid $(21 / 46,45.7 \%)$ and solid $(17 / 46,37.0 \%)$ (data not shown).

As seen in scheme three of Tables II and V, 108/123 rats developed ovarian tumors, with an overall tumor formation rate of $87.8 \%$. There were 25 benign tumors, 27 borderline tumors, 45 ovarian carcinomas (19 LGSC and 26 HGSC) and
11 carcinosarcomas (Figs. 2 and 3); 96 were serous tumors, one was a mucinous tumor and 11 were carcinosarcomas. No carcinosarcomas were found in the $1 \mathrm{mg}$ group, while six and five carcinosarcomas were observed in the 2 and $3 \mathrm{mg}$ groups, respectively (Table V). All 25 benign tumors were cystic tumors; 27 borderline tumors were 20 cystic, one cystic-solid and 6 solid; and 56 malignant tumors were 22 cystic, 21 cystic-solid and 12 solid (data not shown).

As seen in each experimental group (Tables III-V), the tumor formation rate gradually increased with prolonged DMBA exposure time. No tumor formation was observed in the control group. This experiment showed that the 
Table V. Tumor formation rate in different dose and time groups in scheme three.

A, $1.0 \mathrm{mg}$ dose

\begin{tabular}{lcccccc}
\hline Time, days & Total, n/total $(\%)$ & BT, n/total $(\%)$ & BOT, n/total $(\%)$ & OCA, n/total $(\%)$ & OCS, n/total $(\%)$ \\
\hline 60 & $3 / 6(50.0)$ & $2 / 6(33.3)$ & $1 / 6(16.7)$ & $0 / 6$ & $(0.0)$ & $0 / 6$ \\
90 & $8 / 10(80.0)$ & $3 / 10(30.0)$ & $4 / 10(40.0)$ & $1 / 10(10.0)$ & $0 / 10(0.0)$ \\
120 & $8 / 8(100.0)$ & $3 / 8(37.5)$ & $3 / 8(37.5)$ & $2 / 8(25.0)$ & $0 / 8(100.0)$ \\
150 & $9 / 10(90.0)$ & $2 / 10(20.0)$ & $2 / 10(20.0)$ & $5 / 10(50.0)$ & $0 / 10(0.0)$ \\
180 & $9 / 9(100.0)$ & $1 / 9(11.1)$ & $0 / 9(0.0)$ & $8 / 9(88.9)$ & $0 / 9$ & $(0.0)$ \\
Total & $37 / 43(86.0)$ & $11 / 43(25.6)$ & $10 / 43(23.3)$ & $16 / 43(37.2)$ & $0 / 43(0.0)$ \\
\hline
\end{tabular}

$\mathrm{B}, 2.0 \mathrm{mg}$ dose

\begin{tabular}{lccccrr}
\hline Time, days & Total, /total $(\%)$ & BT, n/total $(\%)$ & BOT, n/total $(\%)$ & OCA, n/total $(\%)$ & OCS, n/total $(\%)$ \\
\hline 60 & $5 / 6$ & $(83.3)$ & $4 / 6(66.7)$ & $0 / 6(0.0)$ & $0 / 6$ & $(0.0)$ \\
90 & $8 / 10(80.0)$ & $2 / 10(20.0)$ & $5 / 10(50.0)$ & $1 / 10(10.0)$ & $1 / 6(16.7)$ \\
120 & $10 / 10(100.0)$ & $1 / 10(10.0)$ & $1 / 10(10.0)$ & $6 / 10(60.0)$ & $2 / 10(20.0)$ \\
150 & $10 / 10(100.0)$ & $1 / 10(10.0)$ & $0 / 10(0.0)$ & $7 / 10(70.0)$ & $2 / 10(20.0)$ \\
180 & $6 / 6(100.0)$ & $0 / 6(0.0)$ & $1 / 6(16.7)$ & $4 / 6(66.7)$ & $1 / 6(16.7)$ \\
Total & $39 / 42(92.9)$ & $8 / 42(19.0)$ & $7 / 42(16.7)$ & $18 / 42(42.9)$ & $6 / 42(14.3)$ \\
\hline
\end{tabular}

C, $3.0 \mathrm{mg}$ dose

\begin{tabular}{|c|c|c|c|c|c|}
\hline Time, days & Total, n/total (\%) & BT, n/total (\%) & BOT, n/total (\%) & OCA, n/total (\%) & OCS, n/total (\%) \\
\hline 60 & $6 / 6(100.0)$ & $3 / 6(50.0)$ & $3 / 6 \quad(50.0)$ & $(0.0)$ & $0 / 6 \quad(0.0)$ \\
\hline 90 & 8/9 (88.9) & $1 / 9(11.1)$ & $3 / 9 \quad(33.3)$ & 2/9 (22.2) & $2 / 9(22.2)$ \\
\hline 120 & $6 / 10 \quad(60.0)$ & $2 / 10(20.0)$ & $2 / 10 \quad(20.0)$ & $1 / 10 \quad(10.0)$ & $1 / 10(10.0)$ \\
\hline 150 & 8/9 (88.9) & $0 / 9 \quad(0.0)$ & $1 / 9(11.1)$ & $5 / 9 \quad(55.6)$ & $2 / 9(22.2)$ \\
\hline 180 & 4/4 (100.0) & $0 / 4 \quad(0.0)$ & $1 / 4 \quad(25.0)$ & $3 / 4 \quad(75.0)$ & $0 / 4 \quad(0.0)$ \\
\hline Total & $32 / 38(84.2)$ & 6/38 (15.8) & $10 / 38(26.3)$ & 11/38 (28.9) & $5 / 38(13.2)$ \\
\hline
\end{tabular}

$\mathrm{D}, 0 \mathrm{mg}$ dose

\begin{tabular}{lccccc}
\hline Time, days & Total, $/$ total $(\%)$ & BT, n/total $(\%)$ & BOT, /total $(\%)$ & OCA, n/total $(\%)$ & OCS, n/total $(\%)$ \\
\hline 180 & $0 / 8(0.0)$ & $0 / 8(0.0)$ & $0 / 8(0.0)$ & $0 / 8(0.0)$ & $0 / 8(0.0)$ \\
\hline
\end{tabular}

BT, benign ovarian tumors; BOT, borderline ovarian tumors; OCA, ovarian carcinomas; OCS, ovarian carcinosarcomas.

purse string suture and absorbable gauze affected the early observation of rat ovary. The sutures and the absorbable gauze were completely absorbed in two months and no inflammatory granuloma was seen in the ovaries and tumors.

The histological grades of induced tumors at different doses and time points are also shown in Tables III-V. Ovarian tumor differentiation positively correlated with the dose and induction time in scheme two $(\rho=0.523, P=0.022 ; \rho=0.506$, $\mathrm{P}=0.001$, respectively). The tumor formation rate and the proportion of malignant tumor gradually increased in all three experimental groups with increasing DMBA doses and induction time.
Sizes of tumors and solid components on MR imaging. The MMD of the tumors and solid components are shown in Tables VI and VII and Fig. 5. As seen in Table VI and Fig. 5, the MMD of benign, borderline and malignant tumors were $10.40 \pm 1.99,14.35 \pm 2.29$ and $24.98 \pm 14.80 \mathrm{~mm}$, respectively, in scheme one $(\mathrm{P}=0.005$ and 0.038 for benign and borderline vs. malignant, respectively); 7.86 \pm 2.48 , $10.29 \pm 3.41$ and $15.19 \pm 7.10 \mathrm{~mm}$, respectively, in scheme two $(\mathrm{P}<0.0001$ and $\mathrm{P}=0.001$ for benign and borderline vs. malignant, respectively); and 7.91 $\pm 2.30,9.50 \pm 2.59$ and $15.67 \pm 10.10 \mathrm{~mm}$, respectively, in scheme three $(\mathrm{P}<0.0001$ and $\mathrm{P}=0.002$ for benign and borderline vs. malignant, respectively). 
A

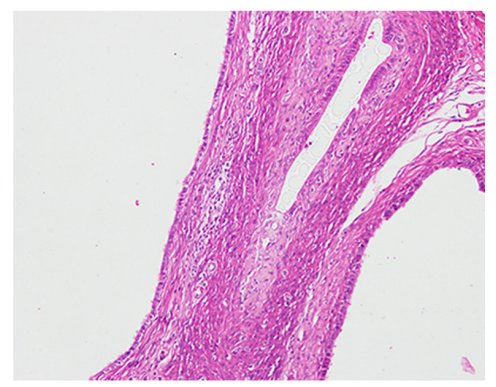

C

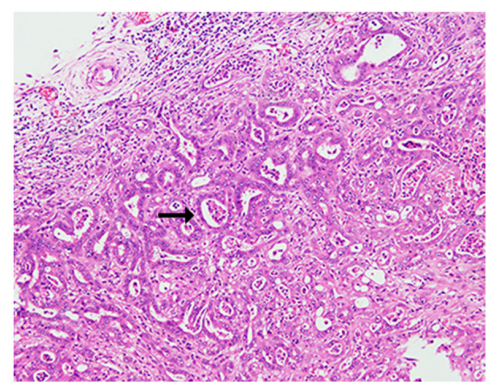

B

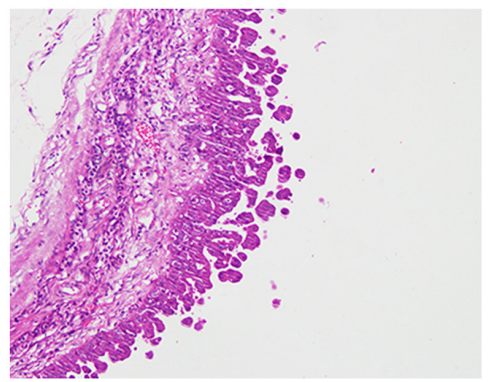

D

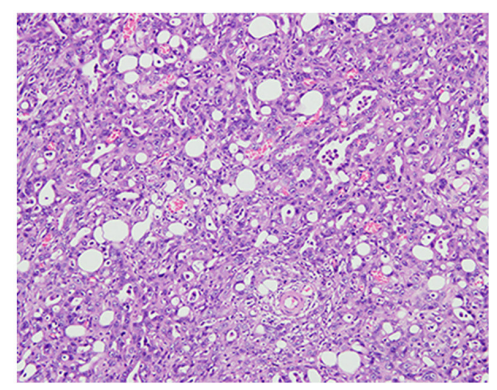

Figure 2. Histopathology of ovarian tumors with different grades (magnification, x200). (A) Ovarian cystadenoma lined by non-stratified columnar cells. (B) Borderline ovarian tumor lined by stratified columnar cells with papillae. (C) Low-grade serous adenocarcinoma with a large number of abnormal cells in the stroma and a relatively regular gland structure (black arrow). (D) High-grade serous adenocarcinoma with pronounced heterogeneity of the stromal cells and incomplete and disordered glandular structure.
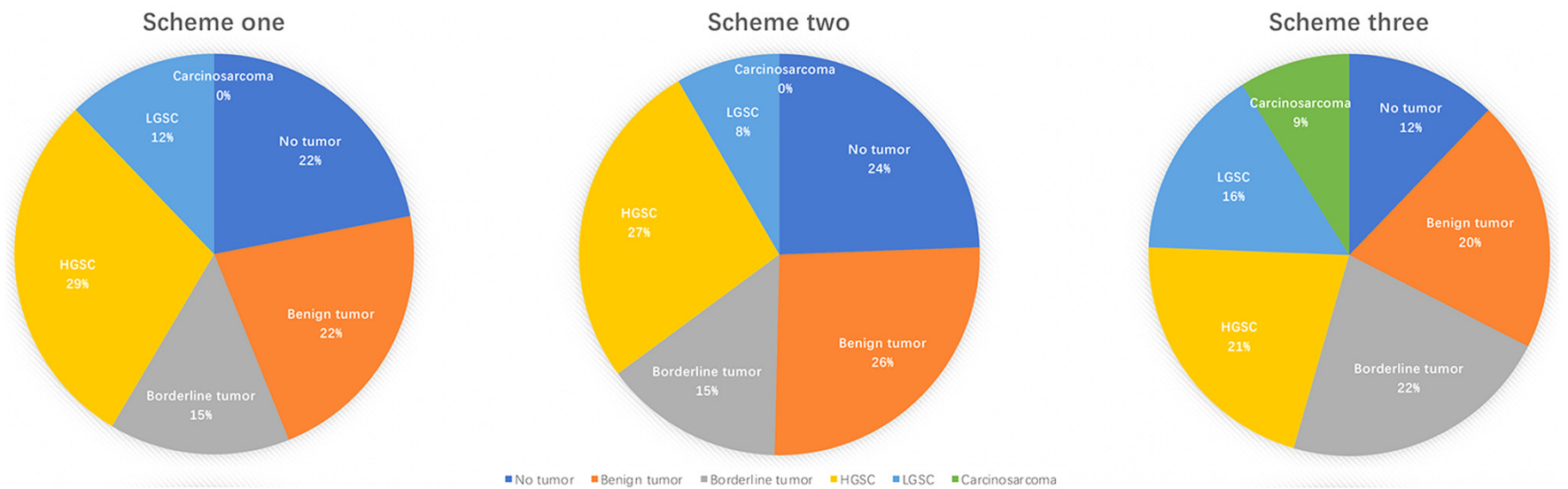

Figure 3. Tumor types in the three schemes. Scheme one included nine benign tumors, six borderline tumors, 12 HGSCs and five LGSCs. There were 34 benign tumors, 19 borderline tumors, 35 HGSCs and 11 LGSCs in scheme two. Scheme three had 25 benign tumors, 27 borderline tumors, 26 HGSCs, 19 LGSCs and 11 carcinosarcomas.

As shown in Table VII and Fig. 5, the MMD of the solid components in benign, borderline and malignant tumors were $0.88 \pm 0.18,1.81 \pm 0.75$ and $13.02 \pm 7.66 \mathrm{~mm}$, respectively, in scheme one; $0.89 \pm 0.19,1.64 \pm 0.62$ and $8.86 \pm 6.89 \mathrm{~mm}$, respectively, in scheme two; and $0.92 \pm 0.36,1.73 \pm 1.33$ and $7.14 \pm 7.71 \mathrm{~mm}$, respectively, in scheme three. All $\mathrm{P}<0.0001$ for benign and borderline vs. malignant in the three schemes. The ovarian tumors induced by this experiment were larger, which are conducive to further research. There was an association between the content of solid components and the degree of malignancy.

\section{Discussion}

A previous study demonstrated that DMBA can induce point mutations that alter the expression of oncogenes and tumor suppressor genes to cause rat ovarian carcinomas (14), which destroy oocytes or early primary follicles, causing pathological changes in the ovaries, and gradually forming tumors (15). Stewart et al (14) induced ovarian tumor formation by encapsulating the ovarian surface with a high concentration of solid DMBA, but the DMBA carrier was a non-absorbable material, which can produce inflammatory granuloma and affect the morphology of the tumors. Therefore, the present study used absorbable gauze as a carrier or a subcapsular injection of a carcinogen to eliminate a foreign body reaction. The experiment showed that the absorbable gauze was completely absorbed in two months, and no obvious inflammatory granuloma was observed.

The mortality rate of the $1.0 \mathrm{mg}$ group was $46.0 \%(23 / 50)$, but the overall mortality rate was as high as $72.7 \%(109 / 150)$ in scheme one. Most rats died of an intestinal obstruction. The 

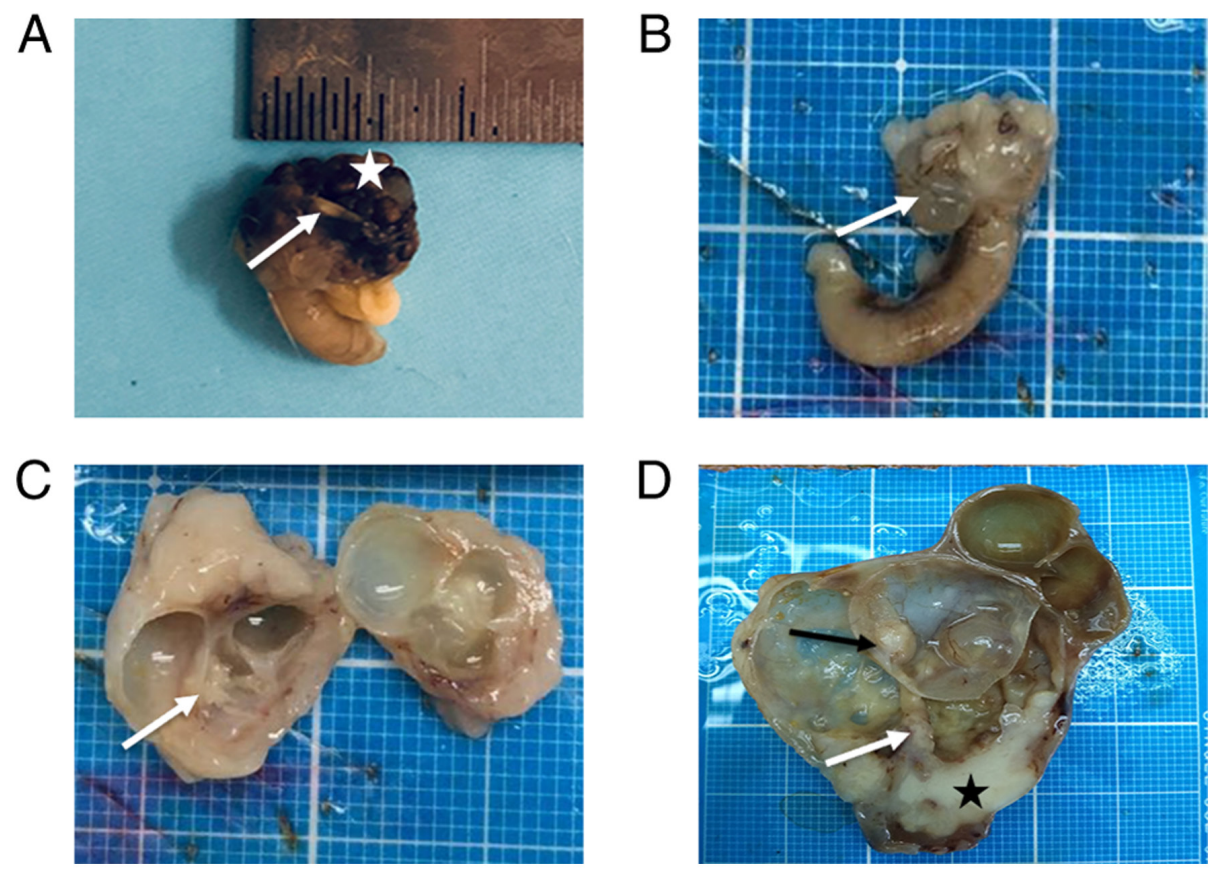

Figure 4. Gross pathological specimens of rat ovarian tumors. (A) Ovary of a Sprague-Dawley rat with ovarian essence (white star) and an ovarian capsule (white arrow). (B) Cystadenoma with a thin wall and clear intracystic fluid (white arrow). (C) Polycystic borderline ovarian tumor with intracystic papillary projections (white arrow). (D) Polycystic ovarian cancer with a thickened septum (white arrow), intracystic papillary projections (black arrow) and a solid component (black star).
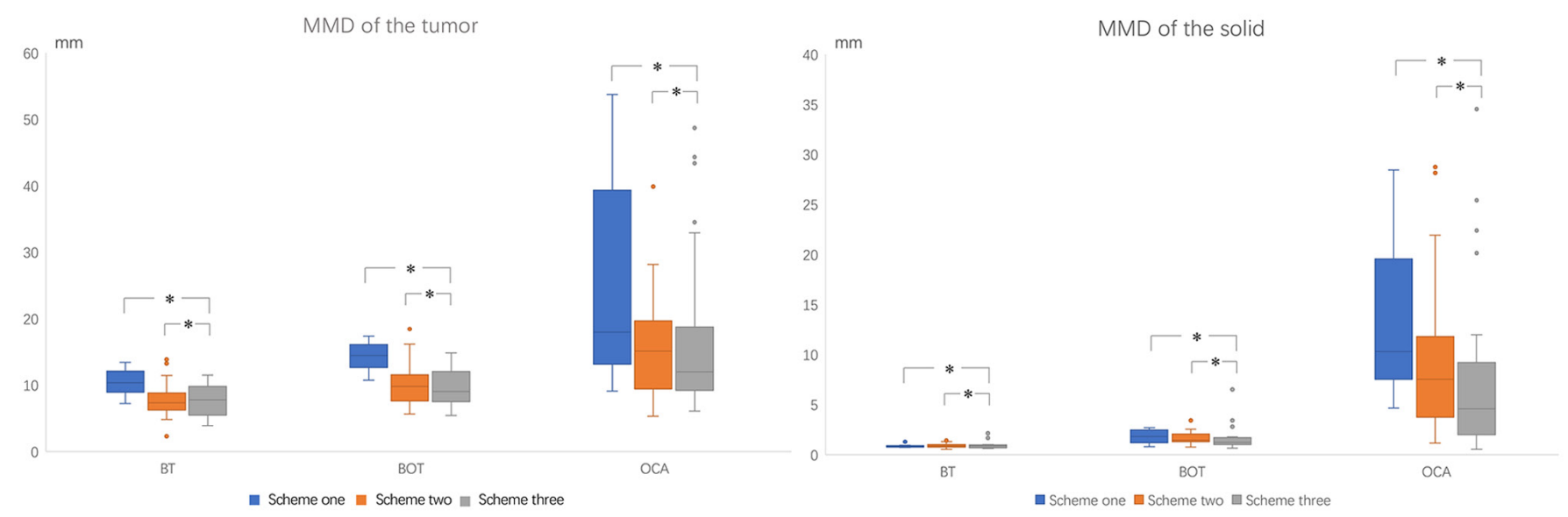

Figure 5. Boxplots showing the MMD of the tumors and the solid components in three schemes. Tumor size and solid component increased progressively with increasing tumor grade; there is no difference between BT and BOT. ${ }^{*} \mathrm{P}<0.05$. MMD, mean maximum diameter; BT, benign tumors; BOT, borderline ovarian tumors; OCA, ovarian carcinomas.

reason for this result might be due to the penetrating ability of DMSO and the loose structure of fat. Carcinogenic DMBA can permeate through the periovary fat, stimulate the intestinal tract and cause intestinal dysfunction (20). In addition, when there is not much fat around the ovary, the sealing effect is poor, and DMBA can leak into the abdominal cavity.

Considering the high mortality rate and possible DMBA leakage in scheme one, we chose to inject DMBA solution under the ovarian capsule in scheme two. The relatively intact and dense ovarian capsule prevented DMBA leakage. The overall mortality rate of rats was only $17.6 \%$ (28/159), which was markedly improved compared with that of scheme one.

A previous study used DMBA-loaded non-absorbable cloth to induce ovarian tumors, and the mortality rate of rats was low (14). To avoid inflammatory granulomas, absorbable gauze was used to carry the melted-to-congealed DMBA in scheme three. The overall mortality rate of rats was $23.6 \%(38 / 161)$, which was lower compared with that of scheme one but higher compared with that of scheme two. With increasing doses, the mortality rates gradually increased. The mortality rate was only $14.0 \%$ (7/50) for the $1.0 \mathrm{mg}$ group and $34.5 \%$ (20/58) for the $3.0 \mathrm{mg}$ group. The rats died at the late stage of the tumor. In this scheme, the tumor formation time was less, and ovarian cancer was more common at three months after induction.

Among the three schemes, the overall tumor formation rate was the highest $(87.7 \%, 108 / 123)$ in scheme three, followed by scheme one $(78.0 \%, 32 / 41)$ and scheme two $(75.6 \%, 99 / 131)$. The higher DMBA content per unit area of absorbable gauze 
Table VI. MMD comparisons of different grade tumors in the three schemes.

\begin{tabular}{|c|c|c|c|c|c|c|c|}
\hline \multirow[b]{2}{*}{ Scheme } & \multicolumn{3}{|c|}{ MMD, mm } & \multirow[b]{2}{*}{ P-value } & \multirow[b]{2}{*}{$\mathrm{P}_{1}$} & \multirow[b]{2}{*}{$\mathrm{P}_{2}$} & \multirow[b]{2}{*}{$\mathrm{P}_{3}$} \\
\hline & BT & BOT & OCA & & & & \\
\hline 1 & $10.40 \pm 1.99$ & $14.35 \pm 2.29$ & $24.98 \pm 14.80$ & 0.0120 & 0.526 & 0.0050 & 0.038 \\
\hline 2 & $7.86 \pm 2.48$ & $10.29 \pm 3.41$ & $15.19 \pm 7.10$ & $<0.0001$ & 0.093 & $<0.0001$ & 0.001 \\
\hline 3 & $7.91 \pm 2.30$ & $9.50 \pm 2.59$ & $15.67 \pm 10.10$ & $<0.0001$ & 0.498 & $<0.0001$ & 0.002 \\
\hline
\end{tabular}

$\mathrm{P}_{1}$, BT vs. BOT; $\mathrm{P}_{2}$, BT vs. OCA; $\mathrm{P}_{3}$, BOT vs. OCA. MMD, mean maximum diameter; BT, benign ovarian tumors; BOT, borderline ovarian tumors; OCA, ovarian carcinomas.

Table VII. MMD comparisons of the solid components of different grade tumors in the three schemes.

\begin{tabular}{|c|c|c|c|c|c|c|c|}
\hline \multirow[b]{2}{*}{ Scheme } & \multicolumn{3}{|c|}{$\mathrm{MMD}, \mathrm{mm}$} & \multirow[b]{2}{*}{ P-value } & \multirow[b]{2}{*}{$\mathrm{P}_{1}$} & \multirow[b]{2}{*}{$\mathrm{P}_{2}$} & \multirow[b]{2}{*}{$\mathrm{P}_{3}$} \\
\hline & BT & BOT & OCA & & & & \\
\hline 1 & $0.88 \pm 0.18$ & $1.81 \pm 0.75$ & $13.02 \pm 7.66$ & $<0.0001$ & 0.773 & $<0.0001$ & $<0.0001$ \\
\hline 2 & $0.89 \pm 0.19$ & $1.64 \pm 0.62$ & $8.86 \pm 6.89$ & $<0.0001$ & 0.557 & $<0.0001$ & $<0.0001$ \\
\hline 3 & $0.92 \pm 0.36$ & $1.73 \pm 1.33$ & $7.14 \pm 7.71$ & $<0.0001$ & 0.646 & $<0.0001$ & $<0.0001$ \\
\hline
\end{tabular}

$\mathrm{P}_{1}$ BT vs. BOT; $\mathrm{P}_{2}$, BT vs. OCA; $\mathrm{P}_{3}$, BOT vs. OCA. MMD, mean maximum diameter; BT, benign ovarian tumors; BOT, borderline ovarian tumors; OCA, ovarian carcinomas.

might explain the different results. The explanation for the slightly lower overall tumor formation rate in scheme two might be that the lower DMBA dose and pinhole leakage led to an insufficient DMBA dose for some rats to form an ovarian tumor. Ovarian carcinosarcomas were induced in scheme three. The high concentration of DMBA might be responsible for the formation of ovarian carcinosarcoma, which is a type of rare ovarian cancer that has not been studied, to the best of our knowledge. Therefore, this model induced by scheme three could be used for investigating carcinosarcoma.

Oncogenesis was not observed in some rats regardless of the scheme used, consistent with previous studies $(13,14)$. At present, there are a few explanations for this phenomenon of the absence of oncogenesis. One theory considers that differences in the times at which DMBA is applied during the ovarian cycle affects the tumor formation rate, but the drug induction time is generally much longer compared with the ovarian cycle time (21). Therefore, this theory has not been widely accepted. Although the carcinogen used in the present experimental model was in contact mainly with the ovary, it might have leaked from pinhole, or been absorbed by the surrounding tissues other than the ovary or carried away from the blood, which resulted in no tumor formation.

Serous tumors accounted for 93.9 and $88.9 \%$ of induced tumors in schemes two and three, respectively. Benign, borderline and malignant tumors were observed in all three schemes, and the main types of malignant tumors were LGSC and HGSC. Since LGSC is a rare and understudied ovarian cancer type, this model would be useful for studying LGSC. In our previous study, DMBA-induced serous tumors were tested for P53 and cyclin D1 and showed positive for
P53 and cyclin D1 (18). Mutations in KRAS and BRAF genes cause continuous expression of cyclin D1 protein that is mainly expressed in serous borderline ovarian tumors and LGSC $(22,23)$. TP53 mutation is much more common in HGSC compared with in LGSC (14).

All benign tumors were cystic tumors, and both borderline and malignant tumors were cystic, cystic-solid and solid. The more solid components the tumor had, the more likely the tumor was to be malignant. A previous study reported that only a small number of serous benign tumors (1.3\%) could form a single small papilla (24), while most serous borderline ovarian tumors had papillae, solid components or were completely solid (25). Li et al (25) showed that the MMD of the solid components was significantly smaller in borderline tumors compared with in ovarian carcinomas. Similar results were obtained in the current study.

Both the DMBA dose and exposure time were positively correlated with ovarian tumor differentiation. Within a certain range, over time and with an increasing dose, the incidence and malignancy of ovarian tumors gradually increased. With an increasing DMBA exposure time, ovarian tumors underwent a gradual oncogenesis process, from benign to borderline to early ovarian carcinomas and advanced ovarian carcinomas, consistent with the occurrence and development of clinical ovarian tumors (26).

The MMD of the tumor and solid components were significantly different between benign and malignant and between borderline and malignant ovarian tumors but not between benign and borderline tumors. The higher the degree of malignancy was, the larger the tumor and solid components were, consistent with the results of a former clinical study (25). 
The morphological manifestations of the induced rat ovarian tumors, including shape, configuration, thickened cyst wall and septal, wall nodule and solid component, were similar to those of human ovarian tumors $(25,27,28)$. The MMD of borderline and malignant tumors were 10.29 and $15.19 \mathrm{~mm}$ in scheme two and 9.50 and $15.67 \mathrm{~mm}$ in scheme three, respectively. The tumors induced by these two schemes were larger compared with those reported in the previous study (14), making the present model favorable for an imaging investigation.

The rat ovarian tumors formed in the three schemes were similar to human ovarian tumors in terms of gross morphology, histological type, pathological appearance, configuration, proportion of various tumors and tumor progression. Each scheme was successful in reducing the foreign body reaction.

Since scheme one had a mortality rate of up to $72.7 \%$ (109/150), it was not an optimal animal model. The overall mortality rate and tumor formation rate of the rats were $17.6 \%(28 / 159)$ and $75.6 \%(99 / 131)$ in scheme two and $23.6 \%(38 / 161)$ and $87.8 \%(108 / 123)$ in scheme three, respectively. Although the tumor formation rate was higher in scheme three compared with in scheme two, the purse string suture of the ovary and absorbable gauze disturbed the early imaging display of the rat ovary. By comparison, the ovarian subcapsular injection of DMBA in scheme two did not affect the early imaging display of the ovary, and MRI could serially demonstrate the gradual decrease in the ovarian parenchyma, the formation of cystic foci and the progressive increase in solid components. The tumor formation rate in the $0.5 \mathrm{mg}$ group was $66.7 \%$ (28/42), which was relatively lower compared with the expected rate $(75 \%, 15 / 20)$. The mortality rate and tumor formation rate were $15.1 \%(8 / 53)$ and $73.3 \%(33 / 45)$ in the $1.0 \mathrm{mg}$ group and $20.0 \%(11 / 55)$ and $86.4 \%$ (38/44) in the $1.5 \mathrm{mg}$ group, respectively. Considering the higher tumor formation rate and the shorter time needed for tumor formation, the optimal dose of scheme two was $1.5 \mathrm{mg}$. Tanaka et al $(29,30)$ performed subcapsular injection of $0.01 \mathrm{ml}$ olive oil with $0.5 \%$ dissolved DMBA and induced the adenocarcinoma in $35-45 \%$ rats after 51 weeks. Liu et al (31) injected $0.05 \mathrm{ml}$ DMBA $(4 \mathrm{mg} / \mathrm{ml})$ and reached an ovarian tumor formation rate of $77.8 \%$ after 20 weeks. Therefore, the tumor formation time of the $1.5 \mathrm{mg}$ group in scheme two was significantly shorter and tumor formation rate was significantly higher in our scheme two model compared with that reported in previous studies (29-31). Furthermore, borderline ovarian tumors appeared in four out of six rats $(66.7 \%)$ after 60 days of DMBA exposure, ovarian carcinomas occurred in three out of eight rats (37.5\%) after 90 days and in seven out of 10 rats $(70 \%)$ after 180 days, and no ovarian carcinosarcomas were found at any time point. This optimal animal model showed the tumor's development process from benign to borderline to malignant successfully in a relatively short period of time. The tumor was relative larger and had similar morphology as human ovarian tumors, suitable for imaging studies of tumor's oncogenesis, development and early detection.

The present study had some limitations. First, the small sample sizes of different time groups might have led to deviations in the research results. Second, a dynamic observation of the whole oncogenic process, such as tumor occurrence, development and metastasis, was not performed. Third, the corresponding gene changes in different histopathological subtypes and differentiation stages were not investigated. In the future, the sample size of different time groups should be increased. In addition, the best time point for the formation of borderline tumors, early and late malignant tumors should be explored. Multi-omics studies on different histopathological subtypes may improve our understanding of the mechanisms underlying the formation and development mechanism of ovarian tumors.

Overall, the ovarian subcapsular injection of $1.5 \mathrm{mg}$ DMBA was the best scheme for the rat ovarian tumor model. This model had a high tumor formation rate. The induced ovarian tumors were large and similar to human ovarian tumors in terms of their gross morphology, histological type, pathological appearance, proportion of various tumors and tumor progression. Therefore, the present rat model is ideal for investigating the occurrence, development and imaging of ovarian tumors.

\section{Acknowledgements}

Not applicable.

\section{Funding}

This work was supported by The National Natural Science Foundation of P.R. China (grant nos. 81471628 and 81971579), The Shanghai Municipal Commission of Science and Technology (grant no. 19411972000) and The Shanghai Municipal Health Commission (grant no. ZK2019B01).

\section{Availability of data and materials}

The datasets used and/or analyzed during the current study are available from the corresponding author on reasonable request.

\section{Authors' contributions}

XYY performed the majority of the experiments. YL and SQC were responsible for experimental guidance. $\mathrm{LW}$ performed pathological analysis. XYY performed the statistical analysis and interpreted the results. XYY and JWQ wrote the manuscript with helpful comments from SQC and YL. SQC, YL, LW and JWQ conceived and designed the project and helped analyze and interpret the results.All authors read and approved the final manuscript.

\section{Ethics approval and consent to participate}

The study was approved by The Institutional Review Board of Jinshan Hospital, Fudan University (Shanghai, China; approval no. 2020-A019-01), and all procedures involving animal studies were in accordance with the Guide for the Care and Use of Laboratory Animals of the National Science and Technology Committee of China.

\section{Patient consent for publication}

Not applicable.

\section{Competing interests}

The authors declare that they have no competing interests. 


\section{References}

1. Bray F, Ferlay J, Soerjomataram I, Siegel RL, Torre LA and Jemal A: Global cancer statistics 2018: GLOBOCAN estimates of incidence and mortality worldwide for 36 cancers in 185 countries. CA Cancer J Clini 68: 394-424, 2018

2. Kurman RJ, Carcangiu ML, Herrington CS and Yong RH (eds.): WHO Classification of Tumours of Female Reproductive Organs. Vol. 6. 4th edition. Lyon, IARC Press, Lyon, pp307, 2014.

3. Prat J: Ovarian carcinomas: Five distinct diseases with different origins, genetic alterations, and clinicopathological features. Virchows Arch 460: 237-249, 2012.

4. Malpica A, Deavers MT, Tornos C, Kurman RJ, Soslow R, Seidman JD, Munsell MF, Gaertner E, Frishberg D and Silva EG: Interobserver and intraobserver variability of a two-tier system for grading ovarian serous carcinoma. Am J Surg Pathol 31: $1168-1174,2007$

5. Shih IeM and Kurman RJ: Ovarian tumorigenesis: A proposed model based on morphological and molecular genetic analysis. Am J Pathol 164: 1511-1518, 2004.

6. Marinaş MC, Mogoş G, Ciurea R and Mogoş DG: EGFR, HER2/neu and Ki67 immunoexpression in serous ovarian tumors. Rom J Morphol Embryol 53: 563-567, 2012.

7. Sieben NL, Macropoulos P, Roemen GM, Kolkman-Uljee SM, Jan Fleuren G, Houmadi R, Diss T, Warren B, Al Adnani M, De Goeij AP, et al: In ovarian neoplasms, BRAF, but not KRAS, mutations are restricted to low-grade serous tumors. J Pathol 202: 336-340, 2004

8. Singer G, Stohr R, Cope L, Dehari R, Hartmann A, Cao DF, Wang TL, Kurman RJ and Shih IeM: Patterns of p53 mutations separate ovarian serous borderline tumors and low- and high-grade carcinomas and provide support for a new model of ovarian carcinogenesis: A mutational analysis with immunohistochemical correlation. Am J Surg Pathol 29: 218-224, 2005.

9. Siegel RL, Miller KD and Jemal A: Cancer statistics, 2019. CA Cancer J Clin 69: 7-34, 2019.

10. Irodi A, Rye T, Herbert K, Churchman M, Bartos C, Mackean M, Nussey F, Herrington CS, Gourley C and Hollis RL: Patterns of clinicopathological features and outcome in epithelial ovarian cancer patients: 35 years of prospectively collected data. BJOG 127: 1409-1420, 2020.

11. Stewart C, Ralyea C and Lockwood S: Ovarian cancer: An integrated review. Semin Oncol Nurs 35: 151-156, 2019.

12. Stakleff KD and Von Gruenigen VE: Rodent models for ovarian cancer research. Int J Gynecol Cancer 13: 405-412, 2003.

13. Hoyer PB, Davis JR, Bedrnicek JB, Marion SL, Christian PJ, Barton JK and Brewer MA: Ovarian neoplasm development by 7,12-dimethylbenz(a)anthracene (DMBA) in a chemically-induced rat model of ovarian failure. Gynecol Oncol 112: 610-615, 2009.

14. Stewart SL, Querec TD, Ochman AR, Gruver BN, Bao R, Babb JS Wong TS, Koutroukides T, Pinnola AD, Klein-Szanto A, et al: Characterization of a carcinogenesis rat model of ovarian preneoplasia and neoplasia. Cancer Res 64: 8177-8183, 2004.

15. Krarup T: Oocyte destruction and ovarian tumorgenesis after direct application of a chemical carcinogen (9:10-dimethyl-1:2-benzanthrene) to the mouse ovary. Int J Cancer 4: 61-75, 1969.

16. Kuwahara I: Experimental induction of ovarian tumors in mice treated with single administration of 7,12-dimethylbenz(a) anthracene, and its histopathological observation. Gan 58 253-266, 1967.

17. Huang Y, Jiang W, Wang Y, Zheng Y, Cong Q and Xu C: Enhanced efficacy and specificity of epithelial ovarian carcinogenesis by embedding a DMBA-coated cloth strip in the ovary of rat. J Ovarian Res 5: 21, 2012.
18. Cai SQ, Li Y,Li YA, Wang L, Zhu J,Zhao SH, Li X and Qiang JW: A rat model of serous borderline ovarian tumors induced by 7,12-dimethylbenz(a)anthracene. Exp Anim 68: 257-265, 2019.

19. Tanaka YO, Okada S, Satoh T, Matsumoto K, Oki A, Saida T, Yoshikawa $\mathrm{H}$ and Minami M: Differentiation of epithelial ovarian cancer subtypes by use of imaging and clinical data: A detailed analysis. Cancer Imaging 16: 3, 2016.

20. Xue M, Ji X, Liang H, Liu Y, Wang B, Sun L and Li W: The effect of fucoidan on intestinal flora and intestinal barrier function in rats with breast cancer. Food Funct 9: 1214-1223, 2018.

21. Nishida T, Sugiyama T, Kataoka A, Ushijima K and Yakushiji M: Histologic characterization of rat ovarian carcinoma induced by intraovarian insertion of a 7,12-dimethylbenz(a)anthracene-coated suture: Common epithelial tumors of the ovary in rats? Cancer 83: 965-970, 1998.

22. Rosenkrantz AB, Sigmund EE, Winnick A, Niver BE, Spieler B, Morgan GR and Hajdu CH: Assessment of hepatocellular carcinoma using apparent diffusion coefficient and diffusion kurtosis indices: Preliminary experience in fresh liver explants. Magn Reson Imaging 30: 1534-1540, 2012.

23. Mori N, Ota H, Mugikura S, Takasawa C, Ishida T, Watanabe G, Tada H, Watanabe M, Takase K and Takahashi S: Luminal-type breast cancer: Correlation of apparent diffusion coefficients with the Ki-67 labeling index. Radiology 274: 66-73, 2015.

24. Seidman JD and Mehrotra A: Benign ovarian serous tumors: A re-evaluation and proposed reclassification of serous 'cystadenomas' and 'cystadenofibromas'. Gynecol Oncol 96: 395-401, 2005.

25. Li YA, Qiang JW, Ma FH, Li HM and Zhao SH: MRI features and score for differentiating borderline from malignant epithelial ovarian tumors. Eur J Radiol 98: 136-142, 2018.

26. Chui MH, Xing D, Zeppernick F, Wang ZQ, Hannibal CG, Frederiksen K, Kjaer SK, Cope L, Kurman RJ, Shih IM, et al: Clinicopathologic and molecular features of paired cases of metachronous ovarian serous borderline tumor and subsequent serous carcinoma. Am J Surg Pathol 43: 1462-1472, 2019.

27. Zhao SH, Qiang JW, Zhang GF, Boyko OB, Wang SJ, Cai SQ and Wang L: MRI appearances of ovarian serous borderline tumor: Pathological correlation. J Magn Reson Imaging 40: 151-156, 2014.

28. Zhao SH, Qiang JW, Zhang GF, Wang SJ, Qiu HY and Wang L: MRI in differentiating ovarian borderline from benign mucinous cystadenoma: Pathological correlation. J Magn Reson Imaging 39: 162-166, 2014.

29. Tanaka T, Kohno H, Tanino M and Yanaida Y: Inhibitory effects of estrogenic compounds, 4-nonylphenol and genistein, on 7,12-dimethylbenz(a)anthracene-induced ovarian carcinogenesis in rats. Ecotoxicol Environ Saf 52: 38-45, 2002.

30. Tanaka T, Kohno H, Suzuki R and Sugie S: Lack of modifying effects of an estrogenic compound atrazine on 7,12-dimethylbenz(a)anthracene-induced ovarian carcinogenesis in rats. Cancer Lett 210: 129-137, 2004.

31. Liu L, Hu Z, Zhang H, Hou Y, Zhang Z, Zhou G and Li B: Vitamin $D$ postpones the progression of epithelial ovarian cancer induced by 7,12-dimethylbenz(a)anthracene both in vitro and in vivo. Onco Targets Ther 9: 2365-2375, 2016

This work is licensed under a Creative Commons Attribution-NonCommercial-NoDerivatives 4.0 International (CC BY-NC-ND 4.0) License. 